\title{
Correction to: Ultrapotassic magmatism in the heyday of the Variscan Orogeny: the story of the Trebíč Pluton, the largest durbachitic body in the Bohemian Massif
}

\author{
Vojtěch Janoušek ${ }^{1}$ (D . Pavel Hanžl ${ }^{1}$ - Martin Svojtka ${ }^{2}$ John M. Hora ${ }^{1}$. Yulia V. Erban Kochergina ${ }^{1} \cdot$ Petr Gadas $^{1}$. \\ František V. Holub ${ }^{3} \cdot$ Axel Gerdes $^{4} \cdot$ Kryštof Verner $^{1} \cdot$ Kristýna Hrdličková $^{1} \cdot$ J. Stephen Daly ${ }^{5} \cdot$ David Buriánek $^{1}$
}

Published online: 13 April 2021

(c) Geologische Vereinigung e.V. (GV) 2021

\section{Correction to: \\ International Journal of Earth Sciences \\ (2020) 109:1767-1810 \\ https://doi.org/10.1007/s00531-020-01872-2}

The authors regret to have inadvertently supplied an erroneous version of the data table displaying recalculated titanite analyses (Electronic Supplementary Material 4; https://link. springer.com/article/10.1007\%2Fs00531-020-01872-2). The authors apologize for any inconvenience caused.

The original article can be found online at https://doi.org/10.1007/ s00531-020-01872-2.

Vojtěch Janoušek

vojtech.janousek@geology.cz

Pavel Hanžl

pavel.hanzl@geology.cz

Martin Svojtka

svojtka@gli.cas.cz

John M. Hora

john.hora@geology.cz

Yulia V. Erban Kochergina

julia.kocergina@geology.cz

Petr Gadas

gadas@sci.muni.cz

Axel Gerdes

gerdes@em.uni-frankfurt.de

Kryštof Verner

krystof.verner@geology.cz

Kristýna Hrdličková

kristyna.hrdlickova@geology.cz
J. Stephen Daly

stephen.daly@ucd.ie

David Buriánek

david.burianek@geology.cz

Czech Geological Survey, Klárov 3, 11821 Prague 1, Czech Republic

2 Institute of Geology of the Czech Academy of Sciences, Rozvojová 269, 16500 Prague 6, Czech Republic

3 Institute of Petrology and Structural Geology, Charles University in Prague, Albertov 6, 12843 Prague 2, Czech Republic

4 Institut für Geowissenschaften, Altenhöferallee 1, 60438 Frankfurt am Main, Germany

5 UCD School of Earth Sciences, Irish Centre for Research in Applied Geosciences (icrag-centre.org), University College Dublin, Belfield, Dublin 4, Ireland 
Table 1 Titanite formulae (atoms per formula unit, apfu) recalculated on the basis of 20 oxygen equivalents; $\mathrm{Fe}^{\mathrm{II}} / \mathrm{Fe}^{\mathrm{III}}$ allocated assuming 12 cations per formula unit (Droop 1987)

\begin{tabular}{|c|c|c|c|}
\hline & $24 / 1323$ & $25 / 1323$ & $30 / \mathrm{K} 0039 \mathrm{C}$ \\
\hline $\mathrm{Nb}_{2} \mathrm{O}_{5}$ & 1.382 & 0.812 & 0.180 \\
\hline $\mathrm{Ta}_{2} \mathrm{O}_{5}$ & b.d.l & 0.422 & b.d.l \\
\hline $\mathrm{SiO}_{2}$ & 29.773 & 29.697 & 30.496 \\
\hline $\mathrm{TiO}_{2}$ & 35.166 & 36.023 & 36.845 \\
\hline $\mathrm{Al}_{2} \mathrm{O}_{3}$ & 1.271 & 1.427 & 1.728 \\
\hline $\mathrm{V}_{2} \mathrm{O}_{3}$ & b.d.l & 0.488 & b.d.l \\
\hline $\mathrm{Fe}_{2} \mathrm{O}_{3}$ & 0.445 & 0.650 & 0.265 \\
\hline $\mathrm{Y}_{2} \mathrm{O}_{3}$ & 0.357 & 0.501 & - \\
\hline $\mathrm{La}_{2} \mathrm{O}_{3}$ & b.d.1 & 0.155 & b.d.l \\
\hline $\mathrm{Ce}_{2} \mathrm{O}_{3}$ & 0.835 & 0.916 & 0.489 \\
\hline $\mathrm{Nd}_{2} \mathrm{O}_{3}$ & 0.773 & 0.814 & 0.272 \\
\hline $\mathrm{CaO}$ & 26.646 & 26.685 & 28.227 \\
\hline $\mathrm{MnO}$ & b.d.l & 0.080 & b.d.l \\
\hline $\mathrm{FeO}$ & 0.000 & 0.000 & 0.000 \\
\hline $\mathrm{SnO}$ & 1.564 & 0.514 & b.d.l \\
\hline $\mathrm{F}$ & 0.230 & 0.248 & 0.297 \\
\hline$\Sigma$ & 98.442 & 99.432 & 98.799 \\
\hline $\mathrm{Nb}$ & 0.085 & 0.049 & 0.011 \\
\hline Тa & 0.000 & 0.015 & 0.000 \\
\hline $\mathrm{Si}$ & 4.060 & 3.984 & 4.019 \\
\hline $\mathrm{Ti}$ & 3.607 & 3.635 & 3.653 \\
\hline $\mathrm{Al}$ & 0.204 & 0.226 & 0.268 \\
\hline V & 0.000 & 0.052 & 0.000 \\
\hline $\mathrm{Fe}^{\mathrm{III}}$ & 0.046 & 0.066 & 0.026 \\
\hline Y & 0.026 & 0.036 & 0.000 \\
\hline $\mathrm{La}$ & 0.000 & 0.008 & 0.000 \\
\hline $\mathrm{Ce}$ & 0.042 & 0.045 & 0.024 \\
\hline $\mathrm{Nd}$ & 0.038 & 0.039 & 0.013 \\
\hline $\mathrm{Ca}$ & 3.893 & 3.835 & 3.986 \\
\hline Mn & 0.000 & 0.009 & 0.000 \\
\hline $\mathrm{Fe}^{\mathrm{II}}$ & 0.000 & 0.000 & 0.000 \\
\hline$\Sigma$ _cations & 12.000 & 12.000 & 12.000 \\
\hline Ti_Y & 3.607 & 3.635 & 3.653 \\
\hline Al_Y & 0.204 & 0.226 & 0.268 \\
\hline $\mathrm{Fe}^{\mathrm{III}}{ }_{-} \mathrm{Y}$ & 0.046 & 0.066 & 0.026 \\
\hline Ta_Y & 0.000 & 0.015 & 0.000 \\
\hline$\Sigma \_Y$ & 3.857 & 3.942 & 3.948 \\
\hline $\mathrm{Ca} \_\mathrm{X}$ & 3.893 & 3.835 & 3.986 \\
\hline $\mathrm{La} \_\mathrm{X}$ & 0.000 & 0.008 & 0.000 \\
\hline Ce_X & 0.042 & 0.045 & 0.024 \\
\hline Nd_X & 0.038 & 0.039 & 0.013 \\
\hline Mn_X & 0.000 & 0.009 & 0.000 \\
\hline $\mathrm{Fe}^{\mathrm{II}} \_\mathrm{X}$ & 0.000 & 0.000 & 0.000 \\
\hline V_X & 0.000 & 0.052 & 0.000 \\
\hline Nb_X & 0.085 & 0.049 & 0.011 \\
\hline Y_X & 0.026 & 0.036 & 0.000 \\
\hline$\overline{\Sigma \_X}$ & 4.083 & 4.074 & 4.033 \\
\hline
\end{tabular}

\section{Reference}

Droop GTR (1987) A general equation for estimating $\mathrm{Fe}^{3+}$ concentrations in ferromagnesian silicates using stoichiometric criteria. Mineral Mag 51:431-435 